\title{
Hypohidrotic Ectodermal Dysplasia with Total Anodontia: A Case Report
}

\author{
K.K. Shashibhushan ${ }^{1}$, Revathy Viswanathan ${ }^{2}$, N. Sathyajith Naik ${ }^{1}$, V.V. Subba Reddy ${ }^{3}$ \\ ${ }^{1}$ MDS, Professor, Department of Pedodontics and Preventive dentistry, College of Dental Sciences, Davangere. \\ ${ }^{2}$ PG student, Department of Pedodontics and Preventive dentistry, College of Dental Sciences, Davangere. \\ ${ }^{3}$ MDS. Professor and Head, Department of Pedodontics and Preventive dentistry, College of Dental Sciences, Davangere.
}

Correspondence:

Department of Pedodontics and Preventive dentistry,

College of Dental Sciences, Davangere

Email: govisrev@rediffmail.com

\begin{abstract}
Ectodermal dysplasia is a hereditary disorder that occurs as a consequence of disturbances in the ectoderm of the developing embryo. The triad of nail dystrophy, alopecia or hypotrichosis and palmoplantar hyperkeratosis is usually accompanied by a lack of sweat glands and a partial or complete absence of primary and/ or permanent dentition. A case report illustrating the prosthetic rehabilitation of a seven year old boy with hypohidrotic ectodermal dysplasia associated with total anodontia is presented.
\end{abstract}

Key words: Complete dentures, ectodermal dysplasia, hypohidrotic, prosthetic rehabilitation, total anodontia. . 


\section{Introduction}

Ectodermal dysplasia is a rare hereditary congenital disease that affects several ectodermal structures. This group of rare inherited disorders is due to the developmental disturbances in the embryonal stage. The outer layer of cells in a developing embryo consists of ectoderm. Structures formed from ectoderm include the teeth, epidermis and appendageal structures, the nervous system and the organs of special senses.

Children with Ectodermal Dysplasia (ED) may have various manifestations of the disease which may or may not involve teeth, skin, hair, nails, sweat glands, sebaceous and salivary glands. Ectodermal dysplasia may be defined as conditions with at least one of the following features (1)-Trichodysplasia, dental defects, onychodysplasia or dishidrosis as well as atleast one sign showing involvement of other ectodermal structure.

It comprises of over 150 different syndromes of unknown pathogenesis (2). Clinical manifestations depend upon the syndrome afflicting the individual. Among the more common syndromes are Ellis Van Creveld syndrome (Chondro Ectodermal Dysplasia), Cranio Ectodermal Dysplasia, Incontinentia Pigmenti, Ectrodactyly Ectodermal Dysplasia- Cleft lip/ Palate syndrome and Rapp-Hodgkin Ectodermal Dysplasia (3). Hypohidrotic ectodermal dysplasia, also called as Christ-SiemensTouraine syndrome or anhidrotic ectodermal dysplasia is the most common of all forms. Its frequency has been reported to be affecting 1-7 per 10,000 live births (4). While other types may show autosomal dominant or recessive trait, hypohidrotic type shows X linked recessive trait. Males are frequently affected severely and heterozygotic females occasionally show minor manifestations (5).

Hypohidrotic Ectodermal Dysplasia (HED) is manifested as triad of defects that includes hypohidrosis, hypotrichosis, hypodontia (6). Patients with HED usually have skin that is soft, thin, dry, either with complete or partial absence of sweat glands. As a result, they cannot perspire normally and may have heat intolerance and hyperplasia. Hair follicles and sebaceous glands are often defective or absent and the hair of the scalp and eyebrows tends to be fine, scanty and blond. Oral manifestations of HED include partial or complete absence of teeth. This can affect both the primary and permanent dentition. The teeth which are present may be conical in shape with large pulps, sometimes hypoplastic and delayed in eruption (7).

Oligodontia or hypodontia leads to an atrophy of the alveolar bone. The permanent dentition may also present with impactions and transpositions (3). For children of preschool age, proper prosthetic treatment is necessary for the function of the stomatognathic system and for psychological development (8).

The prosthodontic management of a seven year old child with HED and total anodontia is described here.

\section{Case Report}

A seven year old male child reported to the department of Pedodontics with the chief complaint of absence of teeth in the mouth. The child presented with features of HED and had been diagnosed much earlier by the physicians.

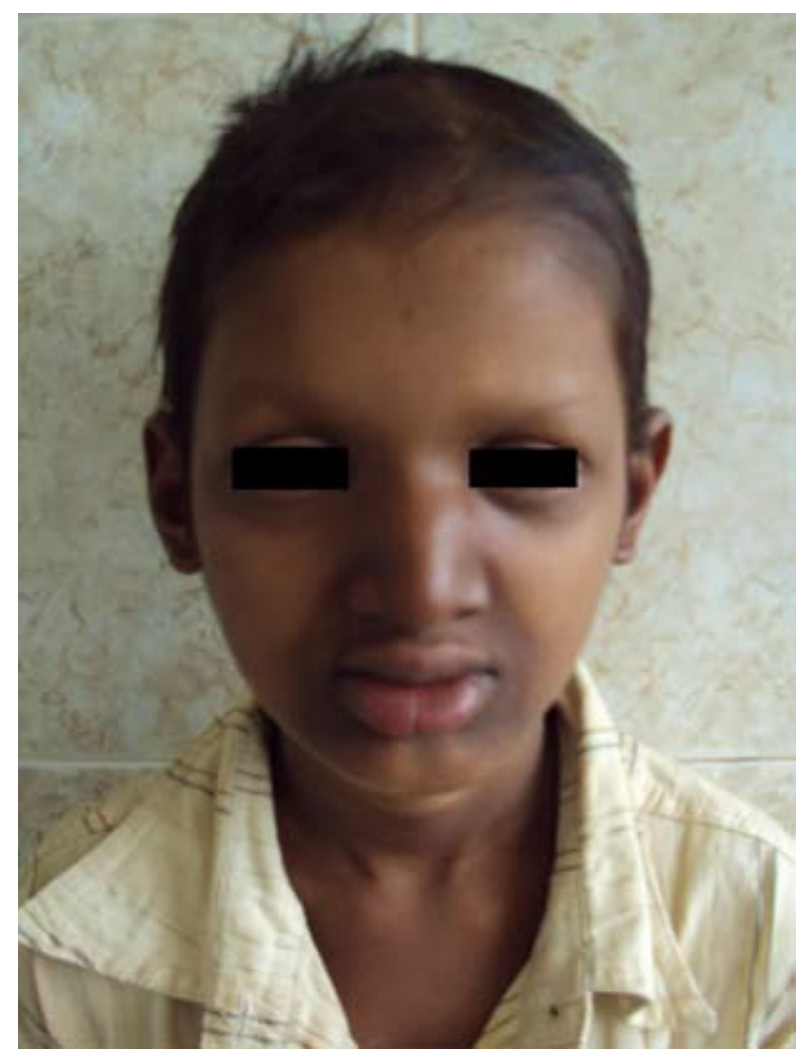

Fig 1. Seven year old patient with Hypohidrotic Ectodermal dysplasia

His hair was blond, fine and sparsely distributed skin smooth and dry with wrinkles. The supra orbital ridges were prominent with sparse eyebrows. Nail examination revealed no abnormality. The lower facial height decreased due to over closed profile, making lips more prominent and protuberant (Fig 1). On intra oral examination, the child had no teeth erupted in the oral cavity. Total anodontia was confirmed in an orthopantomograph (Fig 2). Only basal bone development was seen in the orthopantomograph.

The child was unable to tolerate hot climates and the parents revealed that the child used to wear wet clothes in summer to combat the heat. Examination of the mother showed no abnormality and no tooth was missing. Family history revealed that his younger brother also has HED and was hospitalized because of systemic reasons. No history of the condition among the other members of the family was noted.

Based on the present condition, a treatment plan to fabricate a complete denture was made. The parents and 


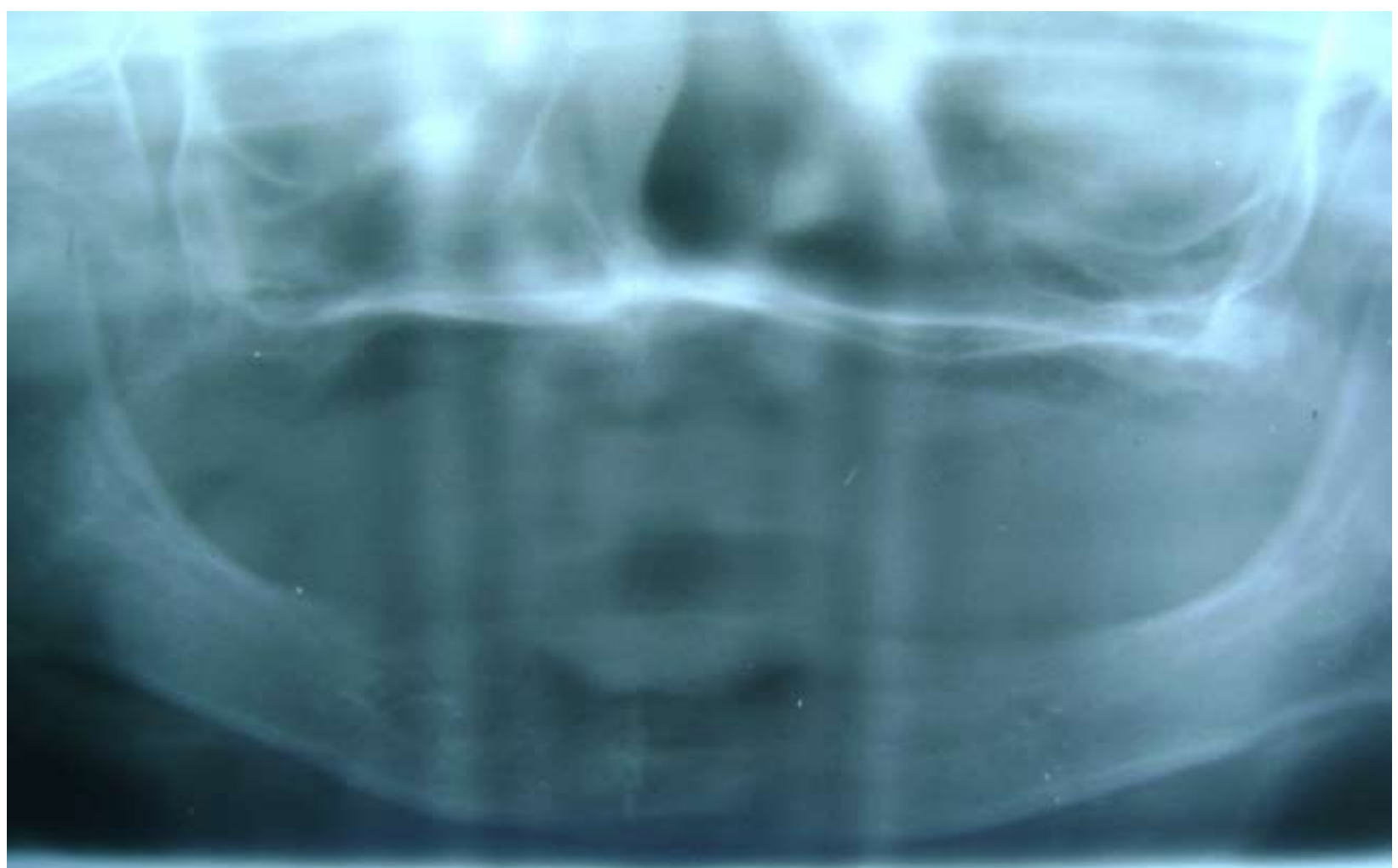

Fig 2. Orthopantomograph showing total anodontia

the patient were explained about the treatment plan. The child was familiarized with various materials, impression trays using "Tell Show Do" technique. Preliminary impressions were recorded with impression compound. The models were obtained and custom trays were fabricated with acrylic resin after spacer adaptation. Border moulding was done with green stick compound. Final impressions were recorded with light body rubber base impression material. Models were obtained and temporary base was constructed with self cure acrylic resin and occlusal rims were fabricated on that. The maxillary rim was adjusted for lip support, phonetics and esthetics. The mandibular rim was adjusted and the centric jaw relation was recorded which was further transferred to the articulator.

Smaller size acrylic teeth were obtained and trimmed according to the esthetic requirements. Try in was done during which the patient and the parents were satisfied. Later the complete dentures were inserted (Fig 3) and instructions were given. It gave the patient a natural appearance.

Recall appointments were scheduled after 24 hours, 1 week, 1 month, during which the patient presented with no problems. Further recall appointments have been scheduled after 3 months.

\section{Discussion}

Optimal treatment for children with hereditary ectodermal dysplasia requires multidisciplinary approach and

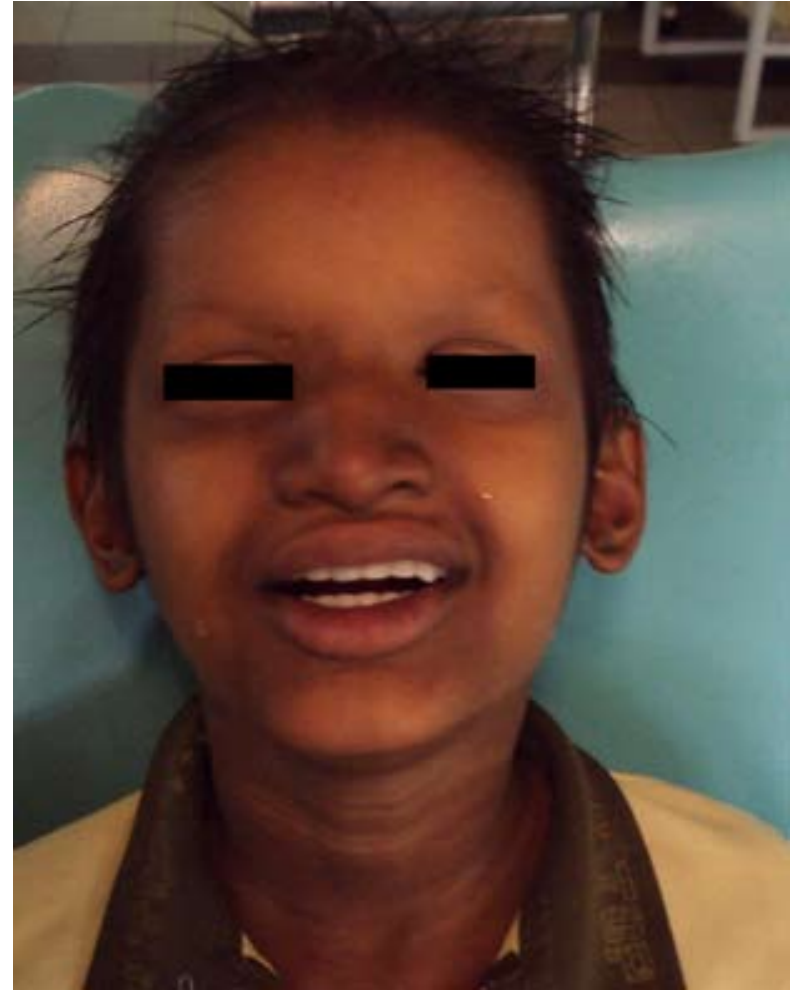

Fig 3. Complete denture insertion

knowledge of behaviour management of pediatric patients. Nowak (9) suggests "Tell Show Do" technique for the child management. 
Usually hypodontia can be managed by removable partial dentures, fixed prosthesis and complete anodontia by complete dentures and implant supported dentures. However the use of implants in children has not been fully investigated and their use should be postponed in children under 13years (10). This is because of implant movement caused by jaw growth. Although, they can be placed, there is no reason to do so as children readily adapt to removable prosthesis with proper preparation and motivation.

Young children with HED not only have difficulties in eating and speaking but can also sense that their appearance is different from others. Hence early rehabilitation is essential. Enabling children with HED to look and act more like their peers through the use of well fitting and functional dentures which are age appropriate will greatly assist in their transitory into school years.

As the child grows, the dentures will have to be modified and replaced because, although the teeth are absent, the basal bone continues to grow. Hence replacements will be needed till the permanent dentition stage after which implant supported dentures may be thought of. Children should be given every opportunity to develop to their fullest potential. The dentist can make a significant contribution to the overall development and wellbeing of a child with HED.

\section{References}

1. Freire-Maia N. Ectodermal dysplasias. Hum Hered. 1971;21:30912.

2. Kere J, Srivastava AK, Montonen O, Zonana J, Thomas N, Ferguson B, et al. X-linked anhidrotic (hypohidrotic) ectodermal dysplasia is caused by mutation in a novel transmembrane protein. Nat Genet. 1996;13:409-16.

3. Borg P, Midtgaard K. Ectodermal dysplasia: report of four cases. ASDC J Dent Child. 1977;44:314-9.

4. Paschos E, Huth KC, Hickel R. Clinical management of hypohidrotic ectodermal dysplasia with anodontia: case report. J Clin Pediatr Dent. 2002;27:5-8.

5. Clarke A. Hypohidrotic ectodermal dysplasia. J Med Genet. 1987;24:659-663.

6. Ramos V, Giebink DL, Fisher JG, Christensen LC. Complete dentures for a child with hypohidrotic ectodermal dysplasia: a clinical report. J Prosthet Dent. 1995;74:329-31.

7. Crawford PJ, Aldred MJ, Clarke A. Clinical and radiographic dental findings in X linked hypohidrotic ectodermal dysplasia. J Med Genet. 1991;28:181-5.

8. Boj JR, Duran von Arx J, Cortada M, Jimenez A, Golobart J. Dentures for a 3-yr-old child with ectodermal dysplasia: case report. Am J Dent. 1993;6:165-7.

9. Nowak AJ. Dental treatment for patients with ectodermal dysplasias. Birth Defects Orig Artic Ser. 1988;24:243-52.

10. Guckes AD, McCarthy GR, Brahim J. Use of endosseous implants in a 3-year-old child with ectodermal dysplasia: case report and 5-year follow-up. Pediatr Dent. 1997;19:282-5. 